\title{
LOCAL-TO-GLOBAL RESULTS IN VARIABLE EXPONENT SPACES
}

\author{
Peter A. HÄstö
}

\begin{abstract}
In this article a new method for moving from local to global results in variable exponent function spaces is presented. Several applications of the method are also given: Sobolev and trace embeddings; variable Riesz potential estimates; and maximal function inequalities in Morrey spaces are derived for unbounded domains.
\end{abstract}

\section{Introduction}

During the last ten years function spaces with variable exponent and related differential equations have attracted a lot of interest, cf. surveys [14, 38]. Apart from interesting theoretical considerations, these investigations were motivated by a proposed application to modeling electrorheological fluids $[2,36]$, and, more recently, an application to image restoration $[1,8]$. In this article we focus on the function space aspect of variable exponent problems. For more information on the PDE aspect see e.g. $[3,4,6,17,19,22,24,31,39]$.

The study of variable exponent function spaces in higher dimensions was initiated in a 1991 article by O. Kováčik and J. Rákosník [26], where basic properties such as reflexivity and Hölder inequalities were obtained. It was followed by only a few articles in the 90s. The rapid expansion of the field started only in the beginning of the current decade with the advent of techniques which allowed one to control the Hardy-Littlewood maximal operator, and through it many other operators.

One way to describe the impediment to progress in the 90s is a lack of a Hölder inequality for the modular, i.e. the integral form of the Lebesgue norm. In a classical Lebesgue space the relation between the modular $\varrho(\cdot)$ and norm $\|\cdot\|$ is very simple:

$$
\|f\|_{L^{p}(\Omega)}=\left(\varrho_{L^{p}(\Omega)}(f)\right)^{\frac{1}{p}} \quad \text { where } \quad \varrho_{L^{p}(\Omega)}=\int_{\Omega}|f(x)|^{p} d x .
$$

In the variable exponent context we retain the form of the modular, but the norm is defined in the spirit of the Luxemburg norm in Orlicz spaces (or Minkowski functional in abstract spaces):

$$
\|u\|_{L^{p(\cdot)}(\Omega)}:=\inf \left\{\lambda>0: \varrho_{L^{(\cdot)}(\Omega)}\left(\frac{u}{\lambda}\right) \leqslant 1\right\} \quad \text { where } \quad \varrho_{L^{p(\cdot)}(\Omega)}(u):=\int_{\Omega}|u(x)|^{p(x)} d x .
$$

Received by the editors July $5,2008$.

2000 Mathematics Subject Classification. 46E30, 46E35.

Key words and phrases. Non-standard growth, variable exponent, Lebesgue space, Sobolev space, Morrey space, Maximal operator, Riesz potential, Sobolev embedding, Hardy inequality, trace embedding.

This research was supported by the Academy of Finland, INTAS and the Emil Aaltonen Foundation. 
Obviously, in this case no functional relationship between norm and modular holds, i.e. $\|u\|_{L^{p(\cdot)}(\Omega)}=F\left(\varrho_{L^{p()}(\Omega)}(u)\right)$ does not hold for any $F$. In particular, we do not get a Hölder inequality for modular from our inequality for the norm.

The major breakthrough came with L. Diening's work [11] which contained the following weak Hölder-type inequality for the modular:

$$
\left(f_{B(x, r)}|f(y)| d y\right)^{p(x)} \lesssim f_{B(x, r)}|f(y)|^{p(y)} d y+1
$$

provided $p$ is bounded away from 1 and $\infty$ and satisfies the local $\log$-Hölder continuity condition

$$
|p(x)-p(y)| \leqslant \frac{c}{\log (e+1 /|x-y|)}
$$

for all $x, y \in \Omega$. Using Diening's result, one can easily prove the boundedness of the maximal operator on bounded sets. However, the additive error prevents us from adding up local estimates to obtain a global result on $\mathbb{R}^{n}$. (Incidentally, the inequality does not hold without the additive term unless $p$ is constant [27].)

The next quest therefore was to prove a global version of the maximal inequality. Diening [11] achieved this only under the additional, unnatural assumption that $p$ is constant outside some ball. However, it did not take long for D. Cruz-Uribe, A. Fiorenza and C. Neugebauer [9] to show that the maximal operator is bounded on $L^{p(\cdot)}\left(\mathbb{R}^{n}\right)$ if the previous assumptions are complemented by a natural decay condition at infinity:

$$
\left|p(x)-p_{\infty}\right| \leqslant \frac{c}{\log (e+|x|)}
$$

for some $p_{\infty}>1, c>0$ and all $x \in \mathbb{R}^{n}$. A. Nekvinda [34] independently obtained an even slightly stronger result; this result is explained in Remark 2.5.

The pattern described for the maximal operator was repeated a great many times for instance with the Riesz potential operator, the sharp maximal operator, fractional maximal operators, etc.: first one proves an easy local result, and then additional, sometimes messy, optimization allows one to prove also the global version.

The purpose of this article is to introduce a simple and convenient method to pass from local to global results. The idea is simply to generalize the following property of the Lebesgue-norm:

$$
\|f\|_{L^{p}\left(\mathbb{R}^{n}\right)}^{p}=\sum_{i}\|f\|_{L^{p}\left(\Omega_{i}\right)}^{p}
$$

for a partition of $\mathbb{R}^{n}$ into measurable sets $\Omega_{i}$. Once the idea is stated, it is almost trivial to carry out, cf. Theorem 2.4. Nevertheless, it proves to be a very powerful tool, as the rest of the article tries to exhibit. Thus we take results by different teams which have only been proven in bounded domains and extend them to unbounded domains. As a simple "toy example" of how the method is applied we prove in the second part of Section 2 a Hardy inequality in unbounded domains using a result in bounded domains from [23].

In Section 5 we reprove the above mentioned boundedness of the maximal operator in $\mathbb{R}^{n}$ in order to introduce in a simple setting some techniques that are then applied in Morrey spaces. Apart from that, the problems treated are based on articles published 
in 2007-08 which had not been solved in the unbounded case, or solved only under additional assumptions. Specifically, the following problems are considered:

Sect. 3: Sobolev inequalities

- in the case when $p$ is not bounded away from 1, generalizing P. Harjulehto and P. Hästö [21]; and

- in trace spaces, generalizing X.-L. Fan [18].

Sect. 4: Embeddings of Riesz potentials with weights, generalizing N.\&S. Samko and B. Vakulov [37].

Sect. 5: The boundedness of the maximal function

- in $L^{p(\cdot)}\left(\mathbb{R}^{n}\right)$, reproving Nekvinda's result [34]; and

- in variable exponent Morrey spaces, generalizing A. Almeida, J. Hasanov and S. Samko [5] and Y. Mizuta and T. Shimomura [32].

Although the proofs get a bit technical in the later section, it is worth pointing out that the original, local case proofs of these results filled a total of about journal 100 pages, and a similar amount could be expected to be needed if the global results were to be obtained by a direct method, case by case.

To conclude the introduction, let us consider perhaps the biggest advance in the theory of variable exponent spaces since Diening's trick. An extrapolation method was introduced by D. Cruz-Uribe, A. Fiorenza, J.M. Martell and C. Pérez [10] which allows us to pass from weighted, constant exponent spaces to variable, unweighted spaces. Since there is a vast literature on weighted, constant exponent Lebesgue spaces, this allowed them to directly derive results on a variety of topics, including the sharp maximal operator, singular integral operators and multipliers. These results are also directly obtained for the case of unbounded domains. Despite the impressive record of their method, it does not work in every case. The main advantages of the method presented in this paper over the extrapolation method from [10] are

- Extrapolation does not allow weights in the variable exponent case (cf. Riesz potentials, Section 4).

- Extrapolation is not easy to adapt to other than the Lebesgue-norm (cf. Morrey spaces, Section 5).

- Extrapolation requires that we know weighted results (e.g. this leads to extraneous assumptions when dealing with multipliers).

- Extrapolation requires that $p^{+}<\infty$, whereas the new method can be extended to cover this case, as well. ${ }^{1}$

Of course, extrapolation also has definite advantages:

- Our method requires a local, variable exponent result to start with.

- Our method requires a non-trivial proof; extrapolation follows by a one-line argument, when all the right elements are in place.

- Our method requires that $p$ is log-Hölder continuous, whereas extrapolation works under the slightly weaker assumption that the maximal operator is bounded.

\footnotetext{
${ }^{1}$ In [10] it is additionally assumed that $p^{-}>1$. It was pointed out by the referee that this condition has been rendered superfluous by the advances of our understanding of the maximal operator in variable exponent Lebesgue spaces.
} 
In view of these aspects, it is fair to say that the methods are complementary: if extrapolation works and gives a sufficiently good result, then it is the method of choice; when this is not the case, the new method is likely to provide an alternative which is still much simpler than a direct proof.

Notation and conventions. The notation $f \lesssim g$ means that $f \leqslant c g$ for some constant $c$, and $f \approx g$ means $f \lesssim g \lesssim f$. By $c$ we denote a generic constant, whose value may change between appearances even within a single line. By $c Q$ we denote a $c$-fold dilate of the cube $Q$. By $f_{A}$ and $f_{A} f d x$ we denote the average integral of $f$ over $A$. The notation $A: X \hookrightarrow Y$ means that $A$ is a continuous embedding from $X$ to $Y$. Omitting the operator, $X \hookrightarrow Y$, means that the identity is a continuous embedding.

By $\Omega \subset \mathbb{R}^{n}$ we denote an open set. A measurable function $p: \Omega \rightarrow[1, \infty)$ is called a variable exponent, and we denote for $A \subset \Omega$

$$
p_{A}^{+}:=\underset{x \in A}{\operatorname{ess} \sup } p(x), \quad p_{A}^{-}:=\underset{x \in A}{\operatorname{essinf}} p(x), \quad p^{+}:=p_{\Omega}^{+} \quad \text { and } \quad p^{-}:=p_{\Omega}^{-} .
$$

We always assume that $p^{+}<\infty$. We will denote by $\mathcal{P}^{\log }(\Omega)$ the class of variable exponents which are log-Hölder continuous, as defined in the introduction.

The variable exponent Lebesgue space $L^{p(\cdot)}(\Omega)$ consists of all measurable functions $u: \Omega \rightarrow \mathbb{R}$ for which $\|f\|_{L^{p(\cdot)}(\Omega)}<\infty$. Equipped with this norm, $L^{p(\cdot)}(\Omega)$ is a Banach space. The variable exponent Lebesgue space is a special case of an Musielak-Orlicz space. For a constant function $p$, it coincides with the standard Lebesgue space.

\section{The local-to-global method}

In this section we introduce the main tool of this article, Theorem 2.4, which allows us to prove global results from local ones.

We need the following result by A. Nekvinda on equivalence of discrete Lebesgue spaces. The space $l^{\left(q_{j}\right)}$ is defined by the modular

$$
\varrho_{l^{\left(q_{j}\right)}}\left(\left(x_{j}\right)\right):=\sum_{j}\left|x_{j}\right|^{q_{j}}
$$

and the norm is defined by $\left\|\left(x_{j}\right)\right\|_{l^{\left(q_{j}\right)}}:=\inf \left\{\lambda>0: \varrho_{l^{\left(q_{j}\right)}}\left(\left(\frac{x_{j}}{\lambda}\right)\right) \leqslant 1\right\}$.

Lemma 2.1 (Theorem 4.3, [33]). Let $\left(q_{j}\right)$ be a sequence in $(1, \infty)$. If there exists $q_{\infty}$ and $c>0$ such that $\left|q_{j}-q_{\infty}\right| \leqslant \frac{c}{\log (e+i)}$, then $l^{\left(q_{j}\right)} \cong l^{q_{\infty}}$.

Definition 2.2. Let $\left(Q_{j}\right)$ be a partition of $\mathbb{R}^{n}$ into equal sized cubes, ordered so that $i>j$ if $\operatorname{dist}\left(0, Q_{i}\right)>\operatorname{dist}\left(0, Q_{j}\right)$. Let $p$ be log-Hölder continuous. We define a partition norm on $L^{p(\cdot)}\left(\mathbb{R}^{n}\right)$ by

$$
\|f\|_{p(\cdot),\left(Q_{j}\right)}:=\|\| f\left\|_{L^{p \cdot(\cdot)}\left(Q_{j}\right)}\right\|_{l^{p_{\infty}}} .
$$

Note that $\|f\|_{p,\left(Q_{j}\right)}=\|f\|_{p}$ if $p$ is a constant, by (1.1). The only essential property of the norm that we need for the next theorem is the following weak relationship between norm and modular:

$$
\min \left\{\varrho_{L^{p(\cdot)}(\Omega)}(f)^{\frac{1}{p^{-}}}, \varrho_{L^{p(\cdot)}(\Omega)}(f)^{\frac{1}{p^{+}}}\right\} \leqslant\|f\|_{L^{p(\cdot)}(\Omega)} \leqslant \max \left\{\varrho_{L^{p(\cdot)}(\Omega)}(f)^{\frac{1}{p^{-}}}, \varrho_{L^{p(\cdot)}(\Omega)}(f)^{\frac{1}{p^{+}}}\right\} .
$$

The proof of this well-known fact follows directly from the definition of the norm. 
Theorem 2.4. If $p \in \mathcal{P}^{\log }\left(\mathbb{R}^{n}\right)$, then $\|f\|_{p(\cdot),\left(Q_{j}\right)} \approx\|f\|_{p(\cdot)}$, where the cubes $\left(Q_{j}\right)$ are as in Definition 2.2.

Proof. Define $q_{j}:=p_{Q_{j}}^{+}$and $q_{\infty}:=p_{\infty}$. Since $p$ is log-Hölder continuous, we conclude that $\left|q_{j}-q_{\infty}\right| \leqslant c \log \left(e+\operatorname{dist}\left(0, Q_{j}\right)\right)^{-1}$. Since the cubes are in increasing order of distance to the origin, there are at most $(2 j)^{n}$ cubes at distance less than $j$ times the side length of $Q_{0}$ from the origin. Hence $\left|q_{j}-q_{\infty}\right| \leqslant \tilde{c} \log (e+j)^{-1}$, so the condition of Lemma 2.1 is satisfied. It follows that

$$
\|f\|_{p(\cdot),\left(Q_{j}\right)} \approx\|\| f\left\|_{L^{p(\cdot)}\left(Q_{j}\right)}\right\|_{l^{\left(q_{j}\right)}} .
$$

The claim that we are trying to prove is homogeneous, and clearly holds when $\|f\|_{p(\cdot)}=0$. Therefore we may assume that $\|f\|_{p(\cdot)}=1$. Then $\|f\|_{p(\cdot),\left(Q_{j}\right)} \lesssim\|f\|_{p(\cdot)}$ follows if we prove that \|\|$f\left\|_{L^{p()}\left(Q_{j}\right)}\right\|_{l^{q_{j}}} \leqslant c$. Since $q_{j}$ is a bounded sequence, this is equivalent to showing that

$$
\varrho_{l^{\left(q_{j}\right)}}\left(\|f\|_{L^{p(\cdot)}\left(Q_{j}\right)}\right) \leqslant c .
$$

Since $\|f\|_{L^{p(\cdot)}\left(Q_{j}\right)} \leqslant\|f\|_{p(\cdot)}=1$, it follows by $(2.3)$ that

$$
\|f\|_{L^{p(\cdot)}\left(Q_{j}\right)}^{p_{Q_{j}}^{+}} \leqslant \varrho_{L^{p(\cdot)}\left(Q_{j}\right)}(f)
$$

Therefore,

$$
\varrho_{l^{\left(q_{j}\right)}}\left(\|f\|_{L^{p(\cdot)}\left(Q_{j}\right)}\right)=\sum_{j=0}^{\infty}\|f\|_{L^{p(\cdot)}\left(Q_{j}\right)}^{q_{j}} \leqslant \sum_{j=0}^{\infty} \varrho_{L^{p()}\left(Q_{j}\right)}(f)=\varrho_{L^{p(\cdot)}\left(\mathbb{R}^{n}\right)}(f)=1 .
$$

To prove the opposite inequality, we set $q_{j}:=p_{Q_{j}}^{-}$and use the same steps, with the other inequality in (2.3).

Remark 2.5. A. Nekvinda has championed the cause of an integral decay condition which is slightly weaker that the log-Hölder decay condition, see e.g. [35]. His condition on $p$ may be stated as the existence of a constant $c>0$ such that

$$
\int_{\left\{p \neq p_{\infty}\right\}} c^{\frac{1}{p(x)-p_{\infty}}} d x<\infty .
$$

In fact, Lemma 2.1 holds also for the discrete analogue of this condition provided the cubes are properly ordered, and thus Theorem 2.4 actually automatically gives slightly stronger results, with Nekvinda's decay condition instead of the log-Hölder decay condition. Hence all the results of this paper work directly under this more general condition. Thus, if one prefers, the class $\mathcal{P}^{\log }(\Omega)$ can be interpreted throughout the article as locally log-Hölder continuous exponents which satisfy Nekvinda's decay condition. 


\section{Applications to Hardy's and Sobolev's inequality}

In this section we present three examples of how the theorem of the previous section can be applied to upgrade local results, proved only on bounded domains, to global results, valid in all of $\mathbb{R}^{n}$. These results involve variable exponent Sobolev spaces, and to state them we need some definitions.

The variable exponent Sobolev space $W^{1, p(\cdot)}(\Omega)$ consists of functions $u \in L^{p(\cdot)}(\Omega)$ whose distributional gradient $\nabla u$ belongs to $L^{p(\cdot)}(\Omega)$. The variable exponent Sobolev space $W^{1, p(\cdot)}(\Omega)$ is a Banach space with the norm

$$
\|u\|_{W^{1, p(\cdot)}(\Omega)}:=\|u\|_{L^{p(\cdot)}(\Omega)}+\|\nabla u\|_{L^{p^{(\cdot)}(\Omega)}} \text {. }
$$

Since the Sobolev norm is just a sum of Lebesgue norms, it is clear that Theorem 2.4 holds for this norm as well. We define the Sobolev space with zero boundary values, $W_{0}^{1, p(\cdot)}(\Omega)$, as the closure of the set of compactly supported $W^{1, p(\cdot)}(\Omega)$-functions with respect to the norm $\|\cdot\|_{W^{1, p(\cdot)(\Omega)}}[20]$.

\section{Hardy's inequality.}

Hardy inequalities have been studied by several authors in the variable exponent setting, e.g. [7, 23, 25, 30]. Here we consider the following version of Hardy's inequality proved by P. Harjulehto, P. Hästö and M. Koskenoja [23]:

Lemma 3.1. Let $\Omega$ be an open and bounded subset of $\mathbb{R}^{n}$. Let $p \in \mathcal{P}^{\log }(\Omega)$ with $1<p^{-} \leqslant p^{+}<\infty$. Assume that there exists a constant $b>0$ such that

$$
\left|B(z, r) \cap \Omega^{c}\right| \geqslant b|B(z, r)|
$$

for every $z \in \partial \Omega$ and $r>0$. Then

$$
\left\|\frac{u}{\delta_{\Omega}}\right\|_{L^{p(\cdot)}(\Omega)} \lesssim\|u\|_{W^{1, p(\cdot)(\Omega)}}
$$

holds for all $u \in W_{0}^{1, p(\cdot)}(\Omega)$. Here $\delta_{\Omega}(z):=\operatorname{dist}(z, \partial \Omega)$.

Using Theorem 2.4 we can easily remove the boundedness restriction.

Theorem 3.2. Lemma 3.1 holds without the assumption that $\Omega$ is bounded.

Proof. Let $u \in W_{0}^{1, p(\cdot)}(\Omega)$. We consider $u$ as a function on $\mathbb{R}^{n}$ by extending it by 0 to $\mathbb{R}^{n} \backslash \Omega$. Let $\left(Q_{j}\right)$ be a partition of $\mathbb{R}^{n}$ into unit cubes which satisfies the condition of Definition 2.2. Let $\Phi_{j}$ be a Lipschitz function with Lipschitz constant 2 which equals 1 in $Q_{j}$ and is supported in $2 Q_{j}$. Then $\Phi_{j} u \in W_{0}^{1, p(\cdot)}\left(2 Q_{j}\right)$ and Lemma 3.1 implies that

$$
\left\|\frac{u}{\delta_{\Omega}}\right\|_{L^{p(\cdot)}\left(Q_{j}\right)} \leqslant\left\|\frac{\Phi_{j} u}{\delta_{\Omega}}\right\|_{L^{(\cdot)}\left(2 Q_{j}\right)} \leqslant\left\|\frac{\Phi_{j} u}{\delta_{\Omega \cap 2 Q_{j}}}\right\|_{L^{p^{(\cdot)}\left(\Omega \cap 2 Q_{j}\right)}} \lesssim\left\|\Phi_{j} u\right\|_{W^{1, p(\cdot)}\left(2 Q_{j}\right)} \lesssim\|u\|_{W^{1, p(\cdot)}\left(2 Q_{j}\right)} .
$$

Next we apply Theorem 2.4 and this inequality:

$$
\left\|\frac{u}{\delta_{\Omega}}\right\|_{L^{p()}(\Omega)} \approx\left(\sum_{j}\left\|\frac{u}{\delta_{\Omega}}\right\|_{L^{p(\cdot)}\left(Q_{j}\right)}^{p_{\infty}}\right)^{1 / p_{\infty}} \lesssim\left(\sum_{j}\|u\|_{W^{1, p()}\left(2 Q_{j}\right)}^{p_{\infty}}\right)^{1 / p_{\infty}}
$$


Then we note that each cube $2 Q_{j}$ can be covered by $3^{n}$ of the cubes $Q_{k}$. Using this and Theorem 2.4 a second time, we conclude that

$$
\left\|\frac{u}{\delta_{\Omega}}\right\|_{L^{(\rho)}(\Omega)} \lesssim\left(\sum_{j}\|u\|_{W^{1, p()}\left(2 Q_{j}\right)}^{p_{\infty}}\right)^{1 / p_{\infty}} \approx\left(\sum_{j}\|u\|_{W^{1, p()}\left(Q_{j}\right)}^{p_{\infty}}\right)^{1 / p_{\infty}} \approx\|u\|_{W^{1, p(\cdot)}\left(Q_{j}\right)} .
$$

\section{Sobolev's inequality in $\mathbb{R}^{n}$.}

Using Riesz' potential and the Hardy-Littlewood maximal function, one can easily prove a Sobolev inequality in $W^{1, p(\cdot)}\left(\mathbb{R}^{n}\right)$. This was done by L. Diening [12]. However, this leads to the extraneous assumption $p^{-}>1$. P. Harjulehto and the author [21] devised a method based on a weak-type estimate to circumvent this problem. Unfortunately, it was not possible to get global results with this method:

Lemma 3.3 (Theorem 1.1, [21]). Suppose that $p \in \mathcal{P}^{\log }(\Omega)$ with $1 \leqslant p(x) \leqslant c<n$ in a bounded open set $\Omega \subset \mathbb{R}^{n}$. Then $\|u\|_{L^{p^{*}()(\Omega)}} \lesssim\|\nabla u\|_{L^{p()}(\Omega)}$ for every $u \in W_{0}^{1, p(\cdot)}(\Omega)$. Here the constant depends only on $n, p$ and $|\Omega|$.

As usual, $p^{*}$ denotes the point-wise Sobolev conjugate exponent, $p^{*}(x):=\frac{n p(x)}{n-p(x)}$. Note that $p^{*}$ is $\log$-Hölder continuous if $p$ is log-Hölder continuous and bounded away from $n$.

Now for the local-to-global trick:

Theorem 3.4. Suppose that $p \in \mathcal{P}^{\log }\left(\mathbb{R}^{n}\right)$ with $1 \leqslant p(x) \leqslant c<n$ in $\mathbb{R}^{n}$. Then $\|u\|_{L^{p^{*} \cdot(\cdot)}\left(\mathbb{R}^{n}\right)} \lesssim\|u\|_{W^{1, p(\cdot)}\left(\mathbb{R}^{n}\right)}$ for every $u \in W^{1, p(\cdot)}\left(\mathbb{R}^{n}\right)$. Here the constant depends only on $n$ and $p$.

Proof. Let $u \in W^{1, p(\cdot)}\left(\mathbb{R}^{n}\right)$. By homogeneity, it suffices to consider the case $\|u\|_{W^{1, p(\cdot)}\left(\mathbb{R}^{n}\right)}=$ 1. Let $\left(Q_{j}\right)$ be a partition of $\mathbb{R}^{n}$ into unit cubes which satisfies the condition of Definition 2.2. Let $\Phi_{j}$ be a Lipschitz function with Lipschitz constant 2 which equals 1 in $Q_{j}$ and is supported in $2 Q_{j}$. Then $\Phi_{j} u \in W_{0}^{1, p(\cdot)}\left(2 Q_{j}\right)$ and Lemma 3.3 implies that

$$
\|u\|_{L^{p^{*}(\cdot)}\left(Q_{j}\right)} \leqslant\left\|\Phi_{j} u\right\|_{L^{p^{*}(\cdot)}\left(2 Q_{j}\right)} \lesssim\left\|\Phi_{j} \nabla u\right\|_{L^{p(\cdot)}\left(2 Q_{j}\right)} \leqslant\|\nabla u\|_{W^{1, p(\cdot)}\left(2 Q_{j}\right)} \cdot
$$

Next we apply Theorem 2.4:

$$
\|u\|_{L^{p^{*}(\cdot)\left(\mathbb{R}^{n}\right)}} \approx\left(\sum_{j}\|u\|_{L^{p^{*}()(}\left(Q_{j}\right)}^{p_{*}^{*}}\right)^{1 / p_{\infty}^{*}} \lesssim\left(\sum_{j}\|u\|_{W^{1, p()}\left(2 Q_{j}\right)}^{p_{*}^{*}}\right)^{1 / p_{\infty}^{*}} \lesssim\left(\sum_{j}\|u\|_{W^{1, p(\cdot)}\left(Q_{j}\right)}^{p^{*}}\right)^{1 / p_{\infty}^{*}} .
$$

In contrast to the case of the Hardy inequality, we here end up with the wrong power after the inequality for using Theorem 2.4: we would want the norm to be raised to the power of $p_{\infty}$ instead of $p_{\infty}^{*}$. However, since $\|u\|_{W^{1, p(\cdot)}\left(Q_{j}\right)} \leqslant\|u\|_{W^{1, p(\cdot)}\left(\mathbb{R}^{n}\right)}=1$ and $p_{\infty} \leqslant p_{\infty}^{*}$, we conclude that $\|u\|_{W^{1, p())}\left(Q_{j}\right)}^{p_{\infty}^{\infty}} \leqslant\|u\|_{W^{1, p(\cdot)}\left(Q_{j}\right)}^{p_{\infty}}$. Then we can use Theorem 2.4 again:

$$
\|u\|_{L^{p^{*}(\cdot)\left(\mathbb{R}^{n}\right)}} \lesssim\left(\sum_{j}\|u\|_{W^{1, p()}\left(Q_{j}\right)}^{p_{\infty}}\right)^{1 / p_{\infty}^{*}} \approx\|u\|_{W^{1, p(c)}\left(\mathbb{R}^{n}\right)}^{p_{\infty} / p^{*}}=1 .
$$




\section{Sobolev embeddings for traces.}

The trace of a function essentially means a restriction of the function to a lower dimensional subset of its original domain of definition. Since Sobolev functions are, a priori, only equivalence classes of measurable functions, some care is needed in making this rigorous.

In the variable exponent Sobolev spaces traces have been studied in [13, 18, 29]. Since $W^{1, p(\cdot)}(\Omega) \hookrightarrow W_{\text {loc }}^{1,1}(\Omega)$, we know by classical theory that $\left.u\right|_{\partial \Omega} \in L_{\text {loc }}^{1}(\partial \Omega)$. X.-L. Fan [18] studied Sobolev embeddings for the traces of Sobolev functions. His Theorem 2.1 reads:

Lemma 3.5. Let $\Omega \subset \mathbb{R}^{n}$ be an open bounded domain with Lipschitz boundary. Suppose that $\|p\|_{W^{1, \gamma(\Omega)}}<\infty$ and $1 \leqslant p^{-} \leqslant p^{+}<n<\gamma$. Then there is a continuous boundary trace embedding $W^{1, p(\cdot)}(\Omega) \hookrightarrow L^{\frac{(n-1) p(\cdot)}{n-p(\cdot)}}(\partial \Omega)$.

X.-L. Fan also gave results in the unbounded case, however, these results were based on a stronger assumption on the domain, which was assumed to satisfy a strong Lipschitz boundary condition.

With our local-to-global result we can easily upgrade to local trace embedding to a global result without the extra assumption on the boundary. In addition, we require only that $\|p\|_{W^{1, \gamma(3 Q)}}$ is uniformly bounded over unit cubes $Q$, whereas Fan needs to assume that $p \in L^{p(\cdot)}\left(\mathbb{R}^{n}\right)$ and $p \in L^{\infty}\left(\mathbb{R}^{n}\right)$. On the other hand, we need the decay condition at infinity. As pointed out in [18, Remark 5.1], this does not follow from the previous assumptions.

Theorem 3.6. Let $\Omega \subset \mathbb{R}^{n}$ be a domain with Lipschitz boundary. Suppose that $\|p\|_{W^{1, \gamma(3 Q \cap \Omega)}}$ is uniformly bounded over unit cubes $Q$, that $p$ satisfies the decay condition, and that $1 \leqslant p^{-} \leqslant p^{+}<n<\gamma$. Then there is a continuous boundary trace embedding $W^{1, p(\cdot)}(\Omega) \hookrightarrow L^{\frac{(n-1) p \cdot(\cdot)}{n-p())}}(\partial \Omega)$.

Proof. Let us denote $p^{\#}(\cdot)=\frac{(n-1) p(\cdot)}{n-p(\cdot)}$. Assume as before that $\|u\|_{W^{1, p(\cdot)}(\Omega)} \leqslant 1$. Let $\left(Q_{i}\right)$ be a partition of $\mathbb{R}^{n}$ as in Definition 2.2. By Theorem 2.4 we then obtain

$$
\begin{aligned}
& \|u\|_{L^{p^{*}(\cdot)(\partial \Omega)}} \approx\left(\sum\|u\|_{L^{\left.p^{*}()\right)\left(\partial \Omega \cap Q_{i}\right)}}^{p_{\infty}^{*}}\right)^{1 / p_{\infty}^{*}} \lesssim\left(\sum\|u\|_{W^{1, p(\cdot)}\left(\Omega \cap Q_{i}\right)}^{p_{\infty}^{*}}\right)^{1 / p_{\infty}^{*}} \\
& \lesssim\left(\sum\|u\|_{W^{1, p(\cdot)}\left(\Omega \cap Q_{i}\right)}^{p_{\infty}}\right)^{1 / p_{\infty}^{\#}} \approx\|u\|_{W^{1, p(\cdot)}(\Omega)}^{p_{\infty} / p_{\infty}^{*}}=1 .
\end{aligned}
$$

\section{Applications to Riesz potentials}

In this section we consider the variable index Riesz potential on weighted Lebesgue spaces with variable exponent. By a weight we mean a measurable, non-negative function. The weighted Lebesgue space is defined by the norm $\|f\|_{L_{\omega}^{p(\cdot)}(\Omega)}:=\left\|f \omega^{1 / p(\cdot)}\right\|_{L^{p(\cdot)}(\Omega)}$. The weighted modular is defined by

$$
\varrho_{L_{\omega}^{p(\cdot)}(\Omega)}(f):=\int_{\Omega}|f(x)|^{p(x)} \omega(x) d x
$$

and it is clear that the following analogue of (2.3) holds:

$$
\min \left\{\varrho_{L_{\omega}^{p())}(\Omega)}(f)^{\frac{1}{p^{-}}}, \varrho_{L_{\omega}^{p^{(\cdot)}}(\Omega)}(f)^{\frac{1}{p^{+}}}\right\} \leqslant\|f\|_{L_{\omega}^{p(\cdot)}(\Omega)} \leqslant \max \left\{\varrho_{L_{\omega}^{p^{(\cdot)}}(\Omega)}(f)^{\frac{1}{p^{-}}}, \varrho_{L_{\omega}^{p(\cdot)}(\Omega)}(f)^{\frac{1}{p^{+}}}\right\} .
$$


We noted previously that Theorem 2.4 depends only on this property of the norm, and hence we conclude that it holds also for weighted Lebesgue spaces with variable exponent.

In a recent article, N.\&S. Samko and B. Vakulov [37] studied mapping properties of a variable Riesz potential in weighted Lebesgue spaces with variable exponent. The potential operator is defined by

$$
I^{\alpha(x)} f(x):=\int_{\mathbb{R}^{n}} \frac{|f(y)|}{|x-y|^{n-\alpha(x)}} d y .
$$

Assume that $p$ and $\alpha$ are $\log$-Hölder continuous. When $\Omega$ is bounded, they proved in $[37$, Theorem A] that

$$
I^{\alpha(x)}: L_{\omega}^{p(\cdot)}(\Omega) \hookrightarrow L_{\omega^{*}}^{p^{*}(\cdot)}(\Omega),
$$

if $\sup \alpha(x) p(x)<n$, where $p^{\#}(x)=\frac{n p(x)}{n-\alpha(x) p(x)}, \omega$ is a weight, and $\omega^{\#}:=\omega^{p^{\#} / p}$. We will here not get into the details of which weights are allowed, and instead refer to [37, Definition 2.2] for further discussion on this. Suffice it to say by way of example that radial weights with appropriate exponents are allowed.

Note that one could equivalently study the operator

$$
I^{\alpha(\cdot)} f(x):=\int_{\mathbb{R}^{n}} \frac{|f(y)|}{|x-y|^{n-\alpha(y)}} d y
$$

where $\alpha(x)$ is replaced by $\alpha(y)$, since $|x-y|^{\alpha(x)} \approx|x-y|^{\alpha(y)}$ by the log-Hölder continuity of $\alpha$ [11, Lemma 3.2].

For unbounded domains, N.\&S. Samko and B. Vakulov needed to assume that $\alpha$ is constant, in which case they proved that

$$
I^{\alpha}: L_{\omega}^{p(\cdot)}\left(\mathbb{R}^{n}\right) \hookrightarrow L_{\omega^{*}}^{p^{\#}(\cdot)}\left(\mathbb{R}^{n}\right),
$$

if $\sup p(x)<\frac{n}{\alpha}$, where the radial weight is controlled by polynomials at 0 and $\infty$. It turns out that there is a good reason that they could not prove that $I^{\alpha(x)}: L_{\omega}^{p(\cdot)}\left(\mathbb{R}^{n}\right) \hookrightarrow$ $L_{\omega^{*}}^{p^{\#} \cdot()}\left(\mathbb{R}^{n}\right)$ : this embedding does not hold in general, as we now show.

Example 4.1. Let $R>2$ and let $\alpha: \mathbb{R}^{n} \rightarrow(0, n)$ be Lipschitz continuous with $\left.\alpha\right|_{B(0,1)} \equiv$ $\alpha_{0}$ and $\left.\alpha\right|_{\mathbb{R}^{n} \backslash B(0, R)} \equiv \alpha_{\infty}$. The exponent $p$ is defined similarly with values $p_{0}$ and $p_{\infty}$. Set $f(x):=|x|^{-\beta} \chi_{\mathbb{R}^{n} \backslash B(0, R)}(x)$. For $x \in B(0,1)$ we find that

$$
I^{\alpha(x)} f(x)=\int_{\mathbb{R}^{n} \backslash B(0, R)} \frac{|y|^{-\beta}}{|x-y|^{n-\alpha_{0}}} d y \approx \int_{R}^{\infty} r^{-\beta-n+\alpha_{0}+n-1} d r=\infty
$$

provided $\alpha_{0} \geqslant \beta$. On the other hand,

$$
\|f\|_{L^{p()}\left(\mathbb{R}^{n}\right)}=\left\||\cdot|^{-\beta}\right\|_{L^{p_{\infty}}\left(\mathbb{R}^{n} \backslash B(0, R)\right)} \approx \int_{R}^{\infty} r^{-\beta p_{\infty}+n-1} d r<\infty
$$

provided $n<\beta p_{\infty}$. Therefore we see that there exists a function $f \in L^{p(\cdot)}\left(\mathbb{R}^{n}\right)$ with $I^{\alpha(x)} f \equiv \infty$ in $B(0,1)$ provided $\alpha_{0} p_{\infty}>n$.

For functions $p$ and $\alpha$ as before and $f=\chi_{B(0,1)}$, similar calculations show that $I^{\alpha(\cdot)} f \notin L^{p^{*}(\cdot)}\left(\mathbb{R}^{n}\right)$ if $\left(\alpha_{0}-\alpha_{\infty}\right) p_{\infty}^{\prime} \geqslant n$. 
In view of the previous example, there is no point in investigating the global behavior of the potentials $I^{\alpha(x)}$ or $I^{\alpha(\cdot)}$, even in the unweighted case. It is, however, possible to obtain a global result which encompasses all the previous results. For this we introduce the potential operator

$$
I^{\alpha, \wedge} f(x):=\int_{\mathbb{R}^{n}} \frac{|f(y)|}{|x-y|^{n-\alpha(x) \wedge \alpha(y)}} d y,
$$

where $a \wedge b$ denotes $\min \{a, b\}$. As was noted before, $|x-y|^{n-\alpha(x) \wedge \alpha(y)} \approx|x-y|^{n-\alpha(x)} \approx$ $|x-y|^{n-\alpha(y)}$ in a bounded domain. Therefore

$$
I^{\alpha, \wedge} f(x) \lesssim \underbrace{\int_{B(x, 1)} \frac{|f(y)|}{|x-y|^{n-\alpha(x)}} d y}_{=: I_{<}^{\alpha(x)} f(x)}+\underbrace{\int_{\mathbb{R}^{n} \backslash B(x, 1)} \frac{|f(y)|}{|x-y|^{n-\alpha(x) \wedge \alpha(y)}} d y}_{=: I_{>}^{\alpha, \wedge} f(x)} .
$$

Let us now investigate how the local-to-global result can be applied to prove the general, variable $\alpha$ result in the global case. It suffices to study the mapping properties of $I_{<}^{\alpha(x)}$ and $I_{>}^{\alpha_{\infty}}$ separately. For the former we apply Theorem 2.4:

Lemma 4.2. Let $p, \alpha \in \mathcal{P}^{\log }\left(\mathbb{R}^{n}\right)$ with $\sup \alpha(x) p(x)<n$. Then

$$
I_{<}^{\alpha(\cdot)}: L_{\omega}^{p(\cdot)}\left(\mathbb{R}^{n}\right) \hookrightarrow L_{\omega^{\#}}^{p^{\#}(\cdot)}\left(\mathbb{R}^{n}\right),
$$

where the weight $\omega$ satisfies the condition of [37, Theorem B].

Proof. Let $\left(Q_{j}\right)$ be a partition of $\mathbb{R}^{n}$ into unit cubes which satisfies the condition of Definition 2.2. For $x \in Q_{j}, I_{<}^{\alpha(x)} f(x)$ is not affected by the values of $f$ outside $3 Q_{j}$. If $0 \in 3 Q_{j}$, then the conditions of $[37$, Theorem A] are satisfied, and we conclude that

$$
\left\|I_{<}^{\alpha(\cdot)} f\right\|_{L_{\omega^{\#}}^{p^{\#} \cdot(\cdot)}\left(Q_{j}\right)} \lesssim\left\|I_{<}^{\alpha(\cdot)}\left(f \chi_{3 Q_{j}}\right)\right\|_{L_{\omega^{\#}}^{p^{\#}(\cdot)}\left(Q_{j}\right)} \lesssim\left\|I_{<}^{\alpha(\cdot)}\left(f \chi_{3 Q_{j}}\right)\right\|_{L_{\omega^{\#}}^{p^{\#} \cdot(\cdot)}\left(3 Q_{j}\right)} \lesssim\|f\|_{L_{\omega}^{p(\cdot)}\left(3 Q_{j}\right)} \cdot
$$

If $0 \notin 3 Q_{j}$, then $d\left(0,3 Q_{j}\right) \geqslant 1$ since the cubes $Q_{j}$ are unit cubes with integer coordinates. Since $\omega$ satisfies the condition of $[37$, Theorem B] one easily checks that the weight is locally constant in the sense that $\omega_{3 Q_{j}}^{+} \leqslant c \omega_{3 Q_{j}}^{-}$, with constant $c$ independent of $j$. Thus (4.3) follows in this case from the unweighted estimate. Now that we have our local estimate in place, we use Theorem 2.4:

$$
\left\|I_{<}^{\alpha(\cdot)} f\right\|_{L_{\omega^{\#}}^{p^{\#} \cdot(\cdot)}\left(\mathbb{R}^{n}\right)} \approx\|\| I_{<}^{\alpha(\cdot)} f\left\|_{L_{\omega^{\#}}^{p^{\#} \cdot(\cdot)}\left(Q_{j}\right)}\right\|_{p_{\infty}^{\#}} \lesssim\|\| f\left\|_{L_{\omega}^{p \cdot(\cdot)}\left(3 Q_{j}\right)}\right\|_{p_{\infty}^{\#}} \leqslant\left(\sum\|f\|_{L_{\omega}^{p \cdot(\cdot)}\left(Q_{j}\right)}^{p_{\infty}^{\#}}\right)^{1 / p_{\infty}^{\#}} .
$$

By homogeneity we may assume that $\|f\|_{L_{\omega}^{p(\cdot)}\left(\mathbb{R}^{n}\right)}=1$, so that $\|f\|_{L_{\omega}^{p(\cdot)}\left(Q_{j}\right)}^{p_{\infty}^{\#}} \leqslant\|f\|_{L_{\omega}^{p(\cdot)}\left(Q_{j}\right)}^{p_{\infty}}$. Thus

$$
\left\|I_{<}^{\alpha(\cdot)} f\right\|_{L_{\omega^{\#}}^{p^{\#}(\cdot)}\left(\mathbb{R}^{n}\right)} \lesssim\left(\sum\|f\|_{L_{\omega}^{p \cdot(\cdot)}\left(Q_{j}\right)}^{p_{\infty}}\right)^{1 / p_{\infty}^{\#}} \approx\|f\|_{\left.L_{\omega}^{p \cdot(\cdot)} \mathbb{R}_{\infty}^{n}\right)}^{p_{\infty} / p_{\infty}^{\#}}=1 .
$$

We now continue with the other part of the Riesz potential, $I_{>}^{\alpha, \wedge}$.

Lemma 4.4. Let $p, \alpha \in \mathcal{P}^{\log }\left(\mathbb{R}^{n}\right)$ with $\sup \alpha(x) p(x)<n$. Then

$$
I_{>}^{\alpha, \wedge}: L_{\omega}^{p(\cdot)}\left(\mathbb{R}^{n}\right) \hookrightarrow L_{\omega^{\#}}^{p^{\#}(\cdot)}\left(\mathbb{R}^{n}\right),
$$

where the weight $\omega$ satisfies the condition of $[37$, Theorem B]. 
Proof. Let us here present a simple proof using the stronger, log-Hölder decay condition instead of the more general condition of Nekvinda (cf. Remark 2.5). It is possible to adapt the proof for the more general condition using the techniques introduced in Section 5 .

The decay condition on $\alpha$ implies that $|x|^{\alpha(x)} \approx|x|^{\alpha_{\infty}}$ for $|x|>1$. Therefore $\mid x-$ $\left.y\right|^{\alpha(x) \wedge \alpha(y)} \lesssim|x-y|^{\alpha_{\infty}}$, since $1<|x-y| \leqslant 2 \max \{|x|,|y|\}$. Hence $I_{>}^{\alpha, \wedge} f \lesssim I_{>}^{\alpha_{\infty}} f$. Since $I_{>}^{\alpha_{\infty}}: L_{\omega}^{p(\cdot)}\left(\mathbb{R}^{n}\right) \hookrightarrow L_{\omega^{*}}^{p^{\#} \cdot(\cdot)}\left(\mathbb{R}^{n}\right)$ by $[37$, Theorem B], we conclude that the same property holds for $I_{>}^{\alpha, \wedge}$.

Combining the previous two lemmas yields:

Corollary 4.5. Let $p, \alpha \in \mathcal{P}^{\log }\left(\mathbb{R}^{n}\right)$ with $\sup \alpha(x) p(x)<n$. Then

$$
I^{\alpha, \wedge}: L_{\omega}^{p(\cdot)}\left(\mathbb{R}^{n}\right) \hookrightarrow L_{\omega^{\sharp}}^{p^{\#}(\cdot)}\left(\mathbb{R}^{n}\right),
$$

where the weight $\omega$ satisfies the condition of $[37$, Theorem B].

\section{Applications to the maximal operator}

Let us start by giving a new proof of the global boundedness of the maximal operator in variable exponent spaces which is much shorter than the original one. It is interesting to note that A. Lerner and C. Pérez [28] have also recently reproved the result of Nekvinda [34]. Their proof is completely different from the one presented here and is based on a general theorem which they prove in quasi-Banach spaces.

For this we start with the following trivial estimate:

$$
M f(x) \leqslant \underbrace{\sup _{r \in(0,1)} f_{B(x, r)}|f(y)| d y}_{=: M_{<} f(x)}+\underbrace{\sup _{r \in[1, \infty)} f_{B(x, r)}|f(y)| d y}_{=: M_{>} f(x)} .
$$

The part $M_{<}$is easily handled by Theorem 2.4, as we see in Corollary 5.3. To deal with the part $M_{>}$we develop a new method.

We need the following lemma, which is due to L. Diening and S. Samko [16]. Their version has $L^{p^{+}}\left(\mathbb{R}^{n}\right)$ in place of $L^{p_{\infty}}\left(\mathbb{R}^{n}\right)$, so a short proof is given here for completeness. Recall that the norm in $X \cap Y$ is given by $\|\cdot\|_{X}+\|\cdot\|_{Y}$.

Lemma 5.1 (cf. Lemma 4.5, [16]). Let $p \in \mathcal{P}^{\log }\left(\mathbb{R}^{n}\right)$. Then

$$
L^{p(\cdot)}\left(\mathbb{R}^{n}\right) \cap L^{\infty}\left(\mathbb{R}^{n}\right) \cong L^{p_{\infty}}\left(\mathbb{R}^{n}\right) \cap L^{\infty}\left(\mathbb{R}^{n}\right) .
$$

Proof. Let $\bar{p}(x):=\min \left\{p(x), p_{\infty}\right\}$. Then $\bar{p}$ is also log-Hölder continuous on $\mathbb{R}^{n}, \bar{p} \leqslant p$, and $\bar{p}_{\infty}=p_{\infty}$. By $\left[15\right.$, Proposition 6.4], $L^{p(\cdot)}\left(\mathbb{R}^{n}\right) \hookrightarrow L^{\bar{p}(\cdot)}\left(\mathbb{R}^{n}\right)$. On the other hand, $\bar{p} \leqslant p \leqslant \infty$, so $L^{\bar{p}(\cdot)}\left(\mathbb{R}^{n}\right) \cap L^{\infty}\left(\mathbb{R}^{n}\right) \hookrightarrow L^{p(\cdot)}\left(\mathbb{R}^{n}\right)$. Combining these embeddings yields the claim. (Incidentally, [15, Proposition 6.4] is written for log-Hölder continuous exponents, but it also holds for exponents satisfying only Nekvinda's condition.)

To control $M_{>}$we need some understanding of the convolution operator. Its boundedness on $L^{p(\cdot)}\left(\mathbb{R}^{n}\right)$ is in general proven using the boundedness of the maximal operator. Since we now want to reprove this fact, we must take a different route. 
Lemma 5.2. Let $p \in \mathcal{P}^{\log }\left(\mathbb{R}^{n}\right)$ and define $\breve{p}(x):=p_{B(x, 1)}^{-}$and $A f(x):=|f|_{B(x, 1)}$. Then $\check{p} \in \mathcal{P}^{\log }(\Omega), \check{p}_{\infty}=p_{\infty}$ and $A: L^{p(\cdot)}\left(\mathbb{R}^{n}\right) \hookrightarrow L^{\check{p}(\cdot)}\left(\mathbb{R}^{n}\right)$.

Proof. It is easy to see that $\check{p}$ satisfies the local log-Hölder condition and that $\check{p}_{\infty}=p_{\infty}$. The log-Hölder decay condition is also easily checked; if one works with Nekvinda's condition, some more effort is needed, see Lemma 5.4.

We start the estimate of $A f(x)$ with a constant exponent Hölder inequality, followed by Young's inequality with exponent $\frac{p(y)}{\grave{p}(x)}$ :

$$
A f(x)^{\check{p}(x)}=\frac{1}{\lambda} f_{B(x, 1)}\left(\lambda^{\frac{1}{\tilde{p}(x)}}|f(y)|\right)^{\check{p}(x)} d y \leqslant \frac{1}{\lambda} f_{B(x, 1)}|f(y)|^{p(y)}+\lambda^{\frac{p(y)}{p(y)-\check{p}(x)}} d y,
$$

where $\lambda \in(0,1)$. For sufficiently small $\lambda, \theta \mapsto \lambda^{1 / \theta}$ is convex on $\left(0, p^{+}\right]$. Thus we have

$$
\lambda^{\frac{p(y)}{p(y)-\tilde{p}(x)}} \leqslant \lambda^{\frac{1}{p(y)-\tilde{p}(x)}} \leqslant \lambda^{\frac{1}{p(y)-p_{\infty} \mid}}+\lambda^{\frac{1}{\Gamma^{\left(p(x)-p_{\infty}\right.}}} .
$$

Integrating the previous estimate now gives

$$
\begin{aligned}
\int_{\mathbb{R}^{n}} A f(x)^{\breve{p}(x)} d x & \leqslant \int_{\mathbb{R}^{n}} \frac{1}{\lambda} f_{B(x, 1)}|f(y)|^{p(y)}+\lambda^{\frac{1}{p(y)-p_{\infty} \mid}}+\lambda^{\frac{1}{\check{p}^{(x)}-p_{\infty} \mid}} d y d x \\
& =\frac{1}{\lambda} \int_{\mathbb{R}^{n}}|f(x)|^{p(x)}+\lambda^{\frac{1}{p(x)-p_{\infty}}}+\lambda^{\frac{1}{\breve{p}(x)-p_{\infty}}} d x
\end{aligned}
$$

which is finite for sufficiently small $\lambda$, by Nekvinda's condition for $p$ and $\check{p}$.

The following corollary is the globalization of Diening's result [11, Theorem 3.5] which says that $M: L^{p(\cdot)}(\Omega) \hookrightarrow L^{p(\cdot)}(\Omega)$ for bounded $\Omega$.

Corollary 5.3. Let $p \in \mathcal{P}^{\log }\left(\mathbb{R}^{n}\right)$ satisfy $1<p^{-} \leqslant p^{+}<\infty$. Then $M: L^{p(\cdot)}\left(\mathbb{R}^{n}\right) \hookrightarrow$ $L^{p(\cdot)}\left(\mathbb{R}^{n}\right)$.

Proof. As noted above, we have $M f \leqslant M_{<} f+M_{>} f$. Therefore we study the operators $M_{<}$and $M_{>}$separately. Let $\left(Q_{i}\right)$ be a partition of $\mathbb{R}^{n}$ as in Definition 2.2. Then, by Theorem 2.4 and the local boundedness of $M_{<}$, we obtain

$$
\left\|M_{<} f\right\|_{L^{p(\cdot)}(\Omega)} \approx\left\|M_{<} f\right\|_{p(\cdot),\left(Q_{j}\right)} \lesssim\|f\|_{p(\cdot),\left(3 Q_{j}\right)} \approx\|f\|_{L^{p \cdot()}(\Omega)} .
$$

For the other part we see start by noting that $M_{>} f \approx M_{>}(A f)$ with $A$ as in Lemma 5.2. From Hölder's inequality we infer that $A f \in L^{\infty}\left(\mathbb{R}^{n}\right)$. Thus, by Lemmas 5.2 and 5.1,

$$
A: L^{p(\cdot)}\left(\mathbb{R}^{n}\right) \hookrightarrow L^{\infty}\left(\mathbb{R}^{n}\right) \cap L^{\check{p}(\cdot)}\left(\mathbb{R}^{n}\right) \cong L^{\infty}\left(\mathbb{R}^{n}\right) \cap L^{p_{\infty}}\left(\mathbb{R}^{n}\right) .
$$

Since $\left\|M_{>} f\right\|_{p_{\infty}} \leqslant\|M f\|_{p_{\infty}} \lesssim\|f\|_{p_{\infty}}$, we conclude that $M_{>}: L^{\infty}\left(\mathbb{R}^{n}\right) \cap L^{p_{\infty}}\left(\mathbb{R}^{n}\right) \hookrightarrow L^{p(\cdot)}\left(\mathbb{R}^{n}\right)$. Thus $M_{>} \approx M_{>} \circ A: L^{p(\cdot)}\left(\mathbb{R}^{n}\right) \hookrightarrow L^{p(\cdot)}\left(\mathbb{R}^{n}\right)$, as required. Combining the estimates for $M_{<}$and $M_{>}$yields the result.

Let us now get back to the technical part of Lemma 5.2, i.e. the case of Nekvinda's decay condition.

Lemma 5.4. Let $p$ satisfy the local log-Hölder condition and Nekvinda's decay condition. Then $\breve{p}(x):=p_{B(x, 1)}^{-}$satisfies the same conditions. 
Proof. It remains only to check Nekvinda's decay condition. Let $\alpha \in(0,1)$ be such that

$$
\int_{\mathbb{R}^{n}} \alpha^{\frac{1}{|p(x)-p \infty|}} d x<\infty
$$

Let $\beta \in(0, \alpha)$; its value will be specified later. Since $\breve{p}(x) \leqslant p(x)$ we conclude that

$$
\int_{\mathbb{R}^{n}} \beta^{\frac{1}{\not{p}(x)-p_{\infty}}} \chi_{\left\{\check{p}(x)>p_{\infty}\right\}}(x) d x \leqslant \int_{\mathbb{R}^{n}} \alpha^{\frac{1}{\left|p(x)-p_{\infty}\right|}} \chi_{\left\{\check{p}(x)>p_{\infty}\right\}}(x) d x<\infty .
$$

Thus it remains to consider points where $\breve{p}(x)<p_{\infty}$. Let $\left(Q_{i}\right)$ be a partition of $\mathbb{R}^{n}$ into unit cubes. Then

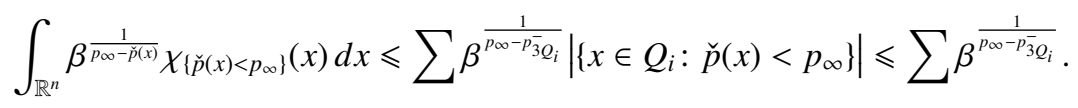

For each $i$ let $x_{i} \in \overline{3 Q_{i}}$ be such that $p\left(x_{i}\right)=p_{3 Q_{i}}^{-}$. If $p\left(x_{i}\right)<p_{\infty}$, then we conclude from the local log-Hölder condition that $p(y)<p_{\infty}-\frac{1}{2}\left(p_{\infty}-p\left(x_{i}\right)\right)$ in a ball $B_{i}$ centered at $x_{i}$ with radius $\exp \left\{-\frac{2 c_{0}}{p_{\infty}-p\left(x_{i}\right)}\right\}$, where $c_{0}$ is the log-Hölder constant. Hence

$$
\alpha^{\frac{2}{p_{\infty}-p\left(x_{i}\right)}} e^{-\frac{2 n c_{0}}{p_{\infty}-p\left(x_{i}\right)}} \approx \alpha^{\frac{2}{p_{\infty}-p\left(x_{i}\right)}}\left|B_{i}\right| \leqslant \int_{B_{i}} \alpha^{\frac{1}{\overline{p(x)-p_{\infty}}}} d x .
$$

Since any given point can occur at most $4^{n}$ times as a point $x_{i}$ we conclude by choosing $\beta=\alpha^{2} e^{-2 n c_{0}}$ that

$$
\sum \beta^{\frac{1}{p_{\infty}-p_{3}^{-} Q_{i}}} \leqslant \sum \alpha^{\frac{2}{p_{\infty}-p\left(x_{i}\right)}} e^{-\frac{2 n c_{0}}{p_{\infty}-p\left(x_{i}\right)}} \leqslant 4^{n} \int_{\mathbb{R}^{n}} \alpha^{\frac{1}{\overline{p(x)-p \infty \mid}}} d x<\infty .
$$

Thus we have shown that $\check{p}$ satisfies Nekvinda's condition with constant $\beta$.

\section{The maximal operator in Morrey spaces.}

Variable exponent Morrey spaces have been studied in [5, 32]. The Morrey space is defined by the modular

$$
\varrho_{L^{p(\cdot),(\cdot)}(\Omega)}(f):=\sup _{z \in \Omega, r>0} r^{-v(z)} \int_{B(z, r)}|f(x)|^{p(x)} d x .
$$

As usual, $\|f\|_{L^{p(-), \cdot(\cdot)}(\Omega)}:=\inf \left\{\lambda>0: \varrho_{L^{p(\cdot),(\cdot)}(\Omega)}(f / \lambda)<1\right\}$. In [5, Lemma 2], it is shown that

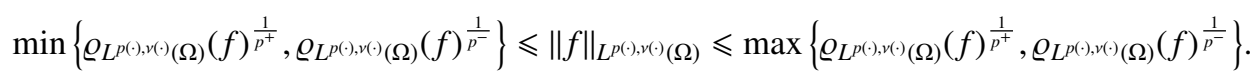

In [5, Lemma 3], an alternative expression for the norm is provided:

$$
\|f\|_{L^{p(\cdot),(\cdot)}(\Omega)}=\sup _{z \in \Omega, r>0}\left\|r^{-\frac{v(z)}{p())}} f\right\|_{L^{p(\cdot)}(B(z, r))} .
$$

For $\left(Q_{j}\right)$ as in Definition 2.2 we define a partition norm on $L^{p(\cdot), v(\cdot)}\left(\mathbb{R}^{n}\right)$ by

$$
\|f\|_{p(\cdot), v(\cdot),\left(Q_{j}\right)}:=\|\| f\left\|_{L^{p(\cdot), \cdot(\cdot)}\left(Q_{j}\right)}\right\|_{l^{p \infty}} .
$$

We now have all the ingredients that were needed in the proof of Theorem 2.4, and may therefore state the following version for Morrey spaces. Being the same, the proof is omitted.

Corollary 5.6. If $p \in \mathcal{P}^{\log }\left(\mathbb{R}^{n}\right)$, then $\|f\|_{p(\cdot), v(\cdot),\left(Q_{j}\right)} \approx\|f\|_{L^{p(\cdot),(\cdot)}(\Omega)}$. 
In [5, Theorem 2] the following result is proven. Theorem 3.3 of [32] contains a different version of the result, with slightly more general spaces, but also more restrictive assumptions on $p$.

Lemma 5.7. Let $\Omega \subset \mathbb{R}^{n}$ be bounded and open, $p \in \mathcal{P}^{\log }(\Omega)$, and let $0 \leqslant v^{-} \leqslant v^{+}<n$. Then $M: L^{p(\cdot), v(\cdot)}(\Omega) \hookrightarrow L^{p(\cdot), v(\cdot)}(\Omega)$.

Using our trick, we now generalize this as follows:

Theorem 5.8. Let $p \in \mathcal{P}^{\log }\left(\mathbb{R}^{n}\right)$ and let $0 \leqslant v^{-} \leqslant v^{+}<n$. Then $M: L^{p(\cdot), v(\cdot)}\left(\mathbb{R}^{n}\right) \hookrightarrow$ $L^{p(\cdot), v(\cdot)}\left(\mathbb{R}^{n}\right)$.

Proof. As before, we have $M f \leqslant M_{<} f+M_{>} f$. Therefore we study the operators $M_{<}$ and $M_{>}$separately. Let $\left(Q_{i}\right)$ be a partition of $\mathbb{R}^{n}$ as in Definition 2.2. Then, by Corollary 5.6,

$$
\left\|M_{<} f\right\|_{L^{p(\cdot), v(\cdot)}\left(\mathbb{R}^{n}\right)} \approx\left\|M_{<} f\right\|_{p(\cdot), v(\cdot),\left(Q_{j}\right)} \lesssim\|f\|_{p(\cdot), v(\cdot),\left(3 Q_{j}\right)} \approx\|f\|_{L^{p(\cdot), v \cdot()}\left(\mathbb{R}^{n}\right)} .
$$

We next consider $M_{>}$. For $B=B(z, r)$ with $r \geqslant 1$ we use Young's inequality:

$$
\begin{aligned}
f_{B}|f(x)| d x & =\int_{B}\left[r^{-\frac{v(z)}{p(x)}}|f(x)|\right] r^{\frac{v(z)}{p(x)}-n} d x \leqslant \int_{B} r^{-v(z)}|f(x)|^{p(x)}+r^{\frac{v(z)-n p(x)}{p(x)-1}} d x \\
& \leqslant r^{-v(z)} \int_{B}|f(x)|^{p(x)} d x+\int_{B} r^{\frac{n(1-p(x))}{p(x)-1}} d x \leqslant \varrho_{L^{p(\cdot), v(\cdot)}\left(\mathbb{R}^{n}\right)}(f)+C .
\end{aligned}
$$

Hence $M_{>} f \in L^{\infty}\left(\mathbb{R}^{n}\right)$. Since $v<n$, it follows that

$$
\sup _{z \in \Omega, r \in(0,1)} r^{-v(z)} \int_{B(z, r)}\left|M_{>} f(x)\right|^{p(x)} d x \leqslant\left(1+\left\|M_{>} f\right\|_{\infty}\right)^{p^{+}} \leqslant c .
$$

Hence we need only consider the Morrey norm over $r \in[1, \infty)$. As in Lemma 5.2 we obtain

$$
\int_{B} A f(x)^{\check{p}(x)} d x \leqslant \frac{1}{\lambda} \int_{2 B}|f(x)|^{p(x)}+\lambda^{\frac{1}{\left|p(x)-p_{\infty}\right|}}+\lambda^{\frac{1}{\not{p}(x)-p_{\infty} \mid}} d x
$$

for $B$ with radius at least 1 , where $A f(x):=|f|_{B(x, 1)}$. Thus we conclude that

$$
r^{-v(z)} \int_{B} A f(x)^{\check{p}(x)} d x \lesssim(2 r)^{-v(z)} \int_{2 B}|f(x)|^{p(x)} d x+C,
$$

and so it follows that $A: L^{p(\cdot), v(\cdot)}\left(\mathbb{R}^{n}\right) \hookrightarrow L^{\check{p}(\cdot), v(\cdot)}\left(\mathbb{R}^{n}\right)$. In view of $(5.5)$, an analogue of Lemma 5.1 holds for Morrey spaces. Hence we conclude as in Corollary 5.3 that $M_{>} f \approx M_{>}(A f)$ and

$$
A: L^{p(\cdot), v(\cdot)}\left(\mathbb{R}^{n}\right) \hookrightarrow L^{\check{p}(\cdot), v(\cdot)}\left(\mathbb{R}^{n}\right) \cap L^{\infty}\left(\mathbb{R}^{n}\right) \cong L^{p_{\infty}, v(\cdot)}\left(\mathbb{R}^{n}\right) \cap L^{\infty}\left(\mathbb{R}^{n}\right) .
$$

Since $\left\|M_{>} f\right\|_{p_{\infty}} \leqslant\|M f\|_{p_{\infty}} \lesssim\|f\|_{p_{\infty}}$, we conclude using (5.5) that $\left\|M_{>} f\right\|_{p_{\infty}, v(\cdot)} \leqslant\|f\|_{p_{\infty}, v(\cdot)}$. Hence

$$
M_{>} L^{p_{\infty}, v(\cdot)}\left(\mathbb{R}^{n}\right) \cap L^{\infty}\left(\mathbb{R}^{n}\right) \hookrightarrow L^{p(\cdot), v(\cdot)}\left(\mathbb{R}^{n}\right) \cap L^{\infty}\left(\mathbb{R}^{n}\right) \hookrightarrow L^{p(\cdot), v(\cdot)}\left(\mathbb{R}^{n}\right)
$$

Thus $M_{>} \approx M_{>} \circ A: L^{p(\cdot), v(\cdot)}\left(\mathbb{R}^{n}\right) \hookrightarrow L^{p(\cdot), v(\cdot)}\left(\mathbb{R}^{n}\right)$, as required.

\section{Acknowledgement}

I would like to thank Lars Diening, Stefan Samko and the anonymous referee for comments and corrections to the original version of this manuscript. 


\section{References}

[1] R. Aboulaich, D. Meskine and A. Souissi: New diffusion models in image processing, Comput. Math. Appl. 56 (2008), no. 4, 874-882.

[2] E. Acerbi and G. Mingione: Regularity results for stationary electro-rheological fluids, Arch. Ration. Mech. Anal. 164 (2002), no. 3, 213-259.

[3] E. Acerbi and G. Mingione: Gradient estimates for the $p(x)$-Laplacean system, J. Reine Angew. Math. 584 (2005), 117-148.

[4] W. Allegretto: Form estimates for the $p(x)$-Laplacean, Proc. Amer. Math. Soc. 135 (2007), no. $7,2177-2185$

[5] A. Almeida, J. Hasanov and S. Samko: Maximal and potential operators in variable exponent Morrey spaces, Georgian Math. J. 15 (2008), no. 2, 195-208.

[6] S. Antontsev and S. Shmarev: Elliptic equations and systems with nonstandard growth conditions: existence, uniqueness and localization properties of solutions, Nonlinear Anal. 65 (2006), no. $4,728-761$.

[7] S. Boza and J. Soria: Weighted Hardy modular inequalities in variable $L^{p}$ spaces for decreasing functions, J. Math. Anal. Appl. 348 (2008), no. 1, 383-388.

[8] Y. Chen, S. Levine and M. Rao: Variable exponent, linear growth functionals in image restoration, SIAM J. Appl. Math. 66 (2006), no. 4, 1383-1406.

[9] D. Cruz-Uribe, A. Fiorenza and C.J. Neugebauer: The maximal function on variable $L^{p}$ spaces, Ann. Acad. Sci. Fenn. Math. 28 (2003), 223-238; 29 (2004), 247-249.

[10] D. Cruz-Uribe, A. Fiorenza, J.M. Martell and C. Pérez: The boundedness of classical operators on variable $L^{p}$ spaces, Ann. Acad. Sci. Fenn. Math. 31 (2006), no. 1, 239-264.

[11] L. Diening: Maximal function on generalized Lebesgue spaces $L^{p(\cdot)}$, Math. Inequal. Appl. 7 (2004), no. 2, 245-253.

[12] L. Diening: Riesz potential and Sobolev embeddings of generalized Lebesgue and Sobolev spaces $L^{p(\cdot)}$ and $W^{k, p(\cdot)}$, Math. Nachr. 263 (2004), no. 1, 31-43.

[13] L. Diening and P. Hästö: Variable exponent trace spaces, Studia Math. 183 (2007), no. 2, 127-141.

[14] L. Diening, P. Hästö and A. Nekvinda: Open problems in variable exponent Lebesgue and Sobolev spaces, FSDONA04 Proceedings (P. Drábek and J. Rákosník (eds.); Milovy, Czech Republic, 2004), 38-58.

[15] L. Diening, P. Hästö and S. Roudenko: Spaces with variable smoothness and integrability, J. Funct. Anal. 256 (2009), no. 6, 1731-1768.

[16] L. Diening and S. Samko: Hardy inequality in variable exponent Lebesgue spaces, Fract. Calc. Appl. Anal. 10 (2007), no. 1, 1-18.

[17] X.-L. Fan: Global $C^{1, \alpha}$ regularity for variable exponent elliptic equations in divergence form, $J$ Differential Equations 235 (2007), no. 2, 397-417.

[18] X.-L. Fan: Boundary trace embedding theorems for variable exponent Sobolev spaces, J. Math. Anal. Appl. 339 (2008), no. 2, 1395-1412.

[19] J. Habermann: Calderón-Zygmund estimates for higher order systems with $p(x)$ growth, Math Z. 258 (2008), no. 2, 427-462.

[20] P. Harjulehto: Variable exponent Sobolev spaces with zero boundary values, Math. Bohem. 132 (2007), no. 2, 125-136.

[21] P. Harjulehto and P. Hästö: Sobolev inequalities for variable exponents attaining the values 1 and $n$, Publ. Mat. 52 (2008), 347-363.

[22] P. Harjulehto, P. Hästö and V. Latvala: Minimizers of the variable exponent, non-uniformly convex Dirichlet energy, J. Math. Pures Appl. (9) 89 (2008), no. 2, 174-197.

[23] P. Harjulehto, P. Hästö and M. Koskenoja: Hardy's inequality in a variable exponent Sobolev space, Georgian Math. J. 12 (2005), no. 3, 431-442.

[24] P. Harjulehto, T. Kuusi, T. Lukkari, N. Marola and M. Parviainen: Harnack's inequality for quasiminimizers with non-standard growth conditions, J. Math. Anal. Appl. 344 (2008), no. 1, $504-520$.

[25] V. Kokilashvili and S. Samko: Maximal and fractional operators in weighted $L^{p(x)}$ spaces, Rev. Mat. Iberoamericana 20(2004), no. 2, 495-517. 
[26] O. Kováčik and J. Rákosník: On spaces $L^{p(x)}$ and $W^{1, p(x)}$, Czechoslovak Math. J. 41(116) (1991), 592-618.

[27] A.K. Lerner: On modular inequalities in variable $L^{p}$ spaces, Arch. Math. (Basel) 85 (2005), no. $6,538-543$.

[28] A.K. Lerner and C. Pérez: A new characterization of the Muckenhoupt $A_{p}$ weights through an extension of the Lorentz-Shimogaki theorem, Indiana Univ. Math. J. 56 (2007), no. 6, 26972722 .

[29] Qiao Liu: Compact trace in weighted variable exponent Sobolev spaces $W^{1, p(x)}\left(\Omega ; v_{0}, v_{1}\right)$, J. Math. Anal. Appl. 348 (2008), no. 2, 760-774.

[30] R.A. Mashiyev, B. Çekiç, F.I. Mamedov and S. Ogras: Hardy's inequality in power-type weighted $L^{p(\cdot)}(0, \infty)$ spaces, J. Math. Anal. Appl. 334 (2007), no. 1, 289-298.

[31] M. Mihăilescu and V. Rădulescu: On a nonhomogeneous quasilinear eigenvalue problem in Sobolev spaces with variable exponent, Proc. Amer. Math. Soc. 135 (2007), no. 9, 2929-2937.

[32] Y. Mizuta and T. Shimomura: Sobolev embeddings for Riesz potentials of functions in Morrey spaces of variable exponent, J. Math. Soc. Japan 60 (2008), no. 2, 583-602.

[33] A. Nekvinda: Equivalence of $\ell^{\left\{p_{n}\right\}}$ norms and shift operators, Math. Inequal. Appl. 5 (2002), no. 4, 711-723.

[34] A. Nekvinda: Hardy-Littlewood maximal operator on $L^{p(x)}\left(\mathbb{R}^{n}\right)$, Math. Inequal. Appl. 7 (2004), no. 2, 255-265.

[35] A. Nekvinda: Maximal operator on variable Lebesgue spaces for almost monotone radial exponent, J. Math. Anal. Appl. 337 (2008), no. 2, 1345-1365.

[36] M. Růžička: Electrorheological fluids: modeling and mathematical theory, Lecture Notes in Mathematics, 1748, Springer-Verlag, Berlin, 2000.

[37] N. Samko, S. Samko and B.G. Vakulov: Weighted Sobolev theorem in Lebesgue spaces with variable exponent, J. Math. Anal. Appl. 335 (2007), no. 1, 560-583.

[38] S. Samko: On a progress in the theory of Lebesgue spaces with variable exponent: maximal and singular operators, Integral Transforms Spec. Funct. 16 (2005), no. 5-6, 461-482.

[39] Q.-H. Zhang: Existence of positive solutions for a class of $p(x)$-Laplacian systems, J. Math. Anal. Appl. 333 (2007), no. 2, 591-603.

Department of Mathematical Sciences, P.O. Box 3000, FI-90014 University of Oulu, FinLAND

E-mail address: peter.hasto@helsinki.fi

URL: http://cc.oulu.fi/ phasto/ 